\title{
How does the size and shape of local populations in China compare to general anthropometric surveys currently used for product design?
}

\author{
Nathan Daniell ${ }^{\mathrm{a}}$, François Fraysse ${ }^{\mathrm{a}, *}$ and Gunther Paul ${ }^{\mathrm{a}}$ \\ ${ }^{a}$ Ergolab, Mawson Institute, University of South Australia, Mawson Lakes SA 5095
}

\begin{abstract}
Anthropometry has long been used for a range of ergonomic applications \& product design. Although products are often designed for specific cohorts, anthropometric data are typically sourced from large scale surveys representative of the general population. Additionally, few data are available for emerging markets like China and India. This study measured 80 Chinese males that were representative of a specific cohort targeted for the design of a new product. Thirteen anthropometric measurements were recorded and compared to two large databases that represented a general population, a Chinese database and a Western database. Substantial differences were identified between the Chinese males measured in this study and both databases. The subjects were substantially taller, heavier and broader than subjects in the older Chinese database. However, they were still substantially smaller, lighter and thinner than Western males. Data from current Western anthropometric surveys are unlikely to accurately represent the target population for product designers and manufacturers in emerging markets like China.
\end{abstract}

Keywords: Anthropometry, Product design, Body size, Body proportions

\section{Introduction}

Anthropometry is used for a range of ergonomic applications \& product design. For example, in automotive ergonomics areas such as occupant package, seat design, restraint system design, crash dummy development and seat comfort assessments are all highly influenced by different anthropometric measurements [1-2]. Anthropometric data used for these applications are often sourced from databases based on large scale anthropometric surveys. Typically these surveys are representative of the general population and may not provide data that accurately represents the targeted consumer. Another issue is the lack of current anthropometric data for emerging markets including India and China [1]. The most recent full body large anthropometric survey completed in China was back in 1988. Product designers for markets such as China are faced with the issue of either using data over 20 years old or applying data from a different subject population. Neither option is favorable if the product is to be designed specifically for the consumer.

The most recent large anthropometric survey of whole body dimensions, completed on the general population of China took place in 1988 [3]. The study included 47 anthropometric dimensions which were measured on 11,164 males aged 18-60 years and 11,150 females aged 18-55 years. One of the largest anthropometric surveys completed on a Western population in the last decade was titled the Civilian American and European Surface Anthropometry Resource (CAESAR) [4]. In total, 2094 males and 2332 females aged 18-65 were measured. Subjects were from the United States of America, The Netherlands and Italy.

*Corresponding author. E-mail: nathan.daniell@unisa.edu.au. Phone: (+61) 883023647 
The aim of this study was to determine how the body size and shape of a specific Chinese cohort differs to the general population of China in 1988 and current Western countries. This information may help determine whether large scale anthropometric surveys in emerging markets are required to better represent the size and shape of people for improved product design.

\section{Method}

Thirteen key anthropometric measurements were taken on 80 Chinese males aged 29-50 years. Sub- jects were sourced from a sample representative of a specific cohort targeted for the design of a new product.

These data were compared to the 1988 China anthropometric database [3] and the US portion of the 2002 CAESAR database [4]. Data from the two databases and this study were compared using the 5 th, 50th and 95th percentiles.

The three studies are summarized in Table 1.

Table 1

Characteristics of the three databases compared in this study.

\begin{tabular}{lcccc}
\hline Name & Year & Subjects (all male) & Age & Population \\
\hline Our study & 2011 & 80 & $29-50$ & Chinese \\
China Standards & 1988 & 11164 & $18-60$ & Chinese \\
CAESAR & 2002 & 2094 & $18-65$ & Western \\
\hline
\end{tabular}

Table 2

Comparison of the $50^{\text {th }}$ percentiles for the three databases assessed in this study.

\begin{tabular}{lcrr}
\hline Measurement & Our study & China (1988) & CAESAR (2002) \\
\hline Stature (mm) & 1727 & 1678 & 1776 \\
Sitting height (mm) & 926 & 908 & 926 \\
Mass (kg) & 74.6 & 59 & 84.7 \\
Buttock to knee length (mm) & 576 & 554 & 617 \\
Waist (umbilicus) circumference (mm) & 894 & 735 & 896 \\
Hip circumference (mm) & 982 & 875 & 1040 \\
Hip breadth (mm) & 340 & 321 & $379 *$ \\
Abdominal depth (mm) & 240 & 212 & $\mathrm{~N} / \mathrm{A}$ \\
Biacromial (shoulder) breadth (mm) & 397 & 422 \\
Acromial (shoulder) height, sitting (mm) & 622 & 375 & 605 \\
Bideltoid breadth (mm) & 470 & 598 & 496 \\
Arm length (mm) & 723 & 431 & 860 \\
Foot length (mm) & 247 & N/A & 268 \\
\hline
\end{tabular}

*Measured sitting; other two studies took measurement standing

\section{Results}

Table 2 compares the $50^{\text {th }}$ percentiles of the three databases assessed in this study.

The Chinese cohort measured in this study differed substantially from both the former Chinese study and the more recent CAESAR study.

When compared to the previous Chinese study, increases can be seen for all measurements with the exception of foot length which remained unchanged. Subjects were on average almost $5 \mathrm{~cm}$ taller and over $15 \mathrm{~kg}$ heavier. Waist circumference and abdominal depth also showed large variations with an increase of $15.9 \mathrm{~cm} \mathrm{(21.6 \% )} \mathrm{and} 2.8 \mathrm{~cm}(13.2 \%)$ respectively, when compared to the 1988 study. Hip circumference also showed a large increase with subjects recording values an average of $10.7 \mathrm{~cm}(12.2 \%)$ greater than the 1988 Chinese study.

Despite the subjects recording values much larger than the 1988 Chinese study, they were still substantially smaller than the US American subjects measured in the CAESAR study. Interestingly, of the 13 measurements compared, acromial height (sitting) 
was the only measurement larger for the Chinese cohort. CAESAR subjects were on average almost 5 $\mathrm{cm}$ taller and just over $10 \mathrm{~kg}$ heavier. The largest difference between the two groups was for arm length with CEASAR subjects having arms $13.7 \mathrm{~cm}$ $(19.0 \%)$ longer than the Chinese subjects measured in this study. Although the Chinese cohort had an almost identical waist circumference to CAESAR subjects, their hip circumference was an average 5.8 cm $(5.9 \%)$ smaller.

While the Chinese cohort measured in this study is larger than the Chinese general population in 1988, they are still considerably smaller than the general population of US American subjects measured in the CAESAR study.

\section{Discussion}

Results suggest that neither the only publicly available 1988 Chinese study, nor the CAESAR study accurately represent the specific cohort in China that was measured for this study. The specific cohort is substantially taller, heavier and broader than that average Chinese male in 1988. Waist and hip circumference also suggest that they carry more subcutaneous fat than the 1988 population.

However, in contrast to this the Chinese cohort is still shorter, lighter and thinner than the US CAESAR population. An exception may be subcutaneous fat stored in the abdominal region as waist circumference was almost identical between the two samples.

These findings have important implications for product design and manufacturing. The size and shape of products are mostly planned based on anthropometric dimensions where the values are sourced from large scale surveys such as CAESAR. This study highlights the differences that may exist between measurements sourced from the large scale surveys and the size and shape of the target populations, which the products are being designed for.
Such differences may result in lower levels of customer satisfaction and could potentially cause injury, due to a poor human-machine fit.

\section{Conclusion}

In conclusion, it has been identified that anthropometric assumptions drawn from previous studies in China and the US were not representative for users of a particular product in China. This poses a number of ergonomic design issues. Without the use of accurate anthropometric data, the ergonomic performance of product design is limited. It would be highly beneficial to conduct further studies comparing the anthropometry of specific cohorts to the anthropometry of the current general population in China, and eventually it will be necessary to conduct a large scale anthropometric survey across the Chinese population. Similar may apply to other emerging markets.

\section{References}

[1] M.P. Reed, S.M. Ebert-Hamilton, M.A. Manary, K.D. Klinich, and L.W. Schneider, A new database of child anthropometry and seated posture for automotive safety applications (Report No. 2005-01-1837). Warrendale, SAE Technical Paper Series, 2005.

[2] D. Kulkarni, S. Ranjan, V. Chitodkar, V. Gurjar, C.V. Ghaisas, and A.V. Mannikar, SIZE INDIA - Anthropometric size measurement of Indian population (Report No. 2011-26-0108). Warrendale, SAE Technical Paper Series, 2011.

[3] China Standards, Human dimensions of Chinese adults (Report No. GB/T 10000-1988). Beijing, Administration of Technology Supervision: People's Republic of China, 1998.

[4] K.M. Robinette, S. Blackwell, H. Daanen, M. Boehmer, S. Fleming, T. Brill, D. Hoeferlin, and D. Burnsides, Civilian Anthropometric and European Surface Anthropometry Resource (CAESAR), Final Report, Volume I: Summary (Report No. AFRL-HE-WP-TR-2002-0169). Ohio, Wright-Patterson AFB, 2002. 